\title{
Using apps to encourage engagement - a new era in medical education
}

\author{
Authors: Ramy Saad ${ }^{A}$ and Emily Farrow ${ }^{B}$
}

\section{Aims}

Any medical lecturer will be familiar with the challenge of maintaining the engagement of their audience. With an increase in the accessibility and fidelity of mobile applications, one avenue for their use is within medical education. A common issue in large lectures is the reluctance of the audience to vocally engage with the speaker - often due to perceived embarrassment in interrupting or a fear of making public errors.

\section{Methods}

We designed a novel teaching course 'The final word' for over 200 MBBS final year students in preparation for their medical finals. Our main focus was to break the fourth wall between audience and lecturer, thus encouraging interaction.

Using a free app (MeeToo), we allowed students to anonymously ask the lecturers and course organisers questions on a live feed. These could be addressed in writing on the public feed or vocally by the speakers.

Over 2 years of running the course we received over 300 feedback forms filled out by students at the end of the 2-day course.

\section{Results}

Over $75 \%$ of students reported they would feel too intimidated or embarrassed to ask a question in a lecture of over 100 people. Sixty-eight per cent of students had downloaded MeeToo and $89 \%$ believe that teaching is improved by the use of such mobile applications. The following year, $89 \%$ felt MeeToo should be used within the medical school setting. Ninety-five per cent felt the course as a whole would improve their performance in MBBS finals and $99 \%$ would recommend the course to a friend.

\section{Conclusion}

There is a consistent fear among medical students of publicly interrupting large lectures to ask questions or make comments. Students responded positively to the use of MeeToo and indicated its use was able to help their education experience. Vitally, they felt medical schools should be implementing this technology.

The use of such applications provides students with relative anonymity and a perceived continuity of the lecture; while they simultaneously engage with speaker. This could be the first step in a more modern and inclusive model for medical education.

Authors: ${ }^{A}$ Guys and St Thomas' NHS Foundation Trust; ${ }^{B}$ East and North Herts NHS Trust 